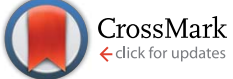

Cite this: RSC Adv., 2016, 6, 112771

Received 21st September 2016 Accepted 21st November 2016

DOI: 10.1039/c6ra23419d

www.rsc.org/advances

\section{Functionalization of fullerene at room temperature: toward new carbon vectors with improved physicochemical properties $\uparrow$}

\author{
Z. Beiranvand, ${ }^{\text {a }}$ A. Kakanejadifard, ${ }^{\text {a }}$ I. S. Donskyi, ${ }^{\text {bc }}$ A. Faghani, ${ }^{\text {b } Z . ~ T u, ~}{ }^{\text {b }}$ A. Lippitz, ${ }^{c}$ \\ P. Sasanpour, ${ }^{\text {de }}$ F. Maschietto, ${ }^{b}$ B. Paulus, ${ }^{b}$ W. E. S. Unger, ${ }^{c}$ R. Haag $^{b}$ and M. Adelit ${ }^{\star a b}$
}

In this work, fullerene has been functionalized with cyanuric chloride at room temperature by a nitrene mediated $[2+1]$ cycloaddition reaction. The adduct after functionalization is inherently in the form of azafulleroid and shows broad UV absorption in the wavelength range of $200-800 \mathrm{~nm}$, as well as photothermal conversion and fluorescence with a high quantum yield.

Fullerene $\left(\mathrm{C}_{60}\right)$ and its functionalized derivatives are interesting candidates for many applications, due to their unique physicochemical properties. ${ }^{1}$ For example, they can be used as antivirals, anticancer compounds, antioxidants as well as drug delivery agents in biomedical science. ${ }^{2,3}$ Poor solubility, low processability and other drawbacks that hamper applications of fullerene have been mostly addressed by covalent and noncovalent functionalization. ${ }^{4}$

In the past few decades a wide range of functionalization approaches has been reported. ${ }^{4,5}$ Nevertheless, a proper tailoring of the distinct physicochemical properties of fullerene remains challenging. ${ }^{6,7}$

UV absorption of fullerene is limited to short wavelengths and therefore it does not show a high photothermal conversion. Furthermore, fullerene and its derivatives exhibit very low fluorescence efficiencies, which render them unavailable for different applications such as bioimaging. ${ }^{8}$ These disadvantages cause fullerenes to be rather inefficient as compared to their one and two dimensional analogues, namely single walled

${ }^{a}$ Faculty of Science, Department of Chemistry, Lorestan University, Khorramabad, Iran ${ }^{b}$ Institut für Chemie und Biochemie, Freie Universität Berlin, Takustrasse 3, Berlin 14195, Germany.E-mail: aadeli@fu-berlin.de; mohadeli@yahoo.com

${ }^{c} B A M$ - Federal Institute for Material Science and Testing, Division of Surface Analysis and Interfacial Chemistry, Unter den Eichen 44-46, 12205 Berlin, Germany

${ }^{d}$ Department of Medical Physics and Biomedical Engineering, School of Medicine, Shahid Beheshti University of Medical Sciences, Tehran, Iran

${ }^{e}$ School of Nanoscience, Institute for Research in Fundamental Sciences (IPM), Tehran, Iran

$\dagger$ Electronic supplementary information (ESI) available. See DOI: 10.1039/c6ra23419d carbon nanotubes and graphene oxide. Due to their defined structure which is a clear advantage over other families of carbon based nanomaterials, fullerene derivatives exhibiting comparable optoelectronic properties could be promising alternatives for different applications including bioimaging ${ }^{9}$ and photothermal therapy.

Nitrene $[2+1]$ cycloaddition reactions and consequently aziridinofullerene-azafulleroid isomerisation is a well-known strategy to obtained nitrogen doped fullerenes with a preserved $\pi$-conjugated system. ${ }^{10}$ This method allows to efficiently functionalize the fullerene cage without affecting the $\mathrm{sp}^{2}$ carbon network, thus leaving the hybridization of the carbon atoms unchanged upon covalent functionalization. ${ }^{11}$ However, the isomerisation reaction often requires relatively high temperatures, which in turn uncompromise the thermal stability of some of the functional groups involved. Recently, we have shown that 2-azido-4,6-dichloro-1,3,5-triazine is a very efficient nitrene precursor and is able to conjugate to carbon based nanomaterials in a $[2+1]$ cycloaddition reaction at room temperature. It was observed that aziridine rings are in the open form and therefore the $\pi$-conjugated system of those nanomaterials is preserved upon functionalization. ${ }^{\mathbf{1 2}}$

In the present work we have successfully extended this strategy for the functionalization of fullerenes. In situ reaction between sodium azide, 2,4,6-trichloro-1,3,5-triazine and fullerene at room temperature results in triazine-functionalized fullerene (Full-Trz) in the azafulleroid form with unusual optoelectronic properties (Scheme 1).

The energy path of the cycloaddition reaction shown in Scheme 1 was investigated computationally by means of a CI$\mathrm{NEB}^{13,14}$ calculation. The latter study revealed that the reaction lacks of an activation energy, hence confirming that the functionalization is energetically favoured and is likely to occur, even at room temperature (Fig. S10†).

The X-ray photoelectron spectroscopy (XPS) spectra of FullTrz revealed the presence of $\mathrm{C}, \mathrm{N}$ and $\mathrm{Cl}$ atoms, proving the conjugation of triazine groups to fullerene (Fig. S1†). In the C 1s peak spectra, in addition to the peak for carbon atoms of 


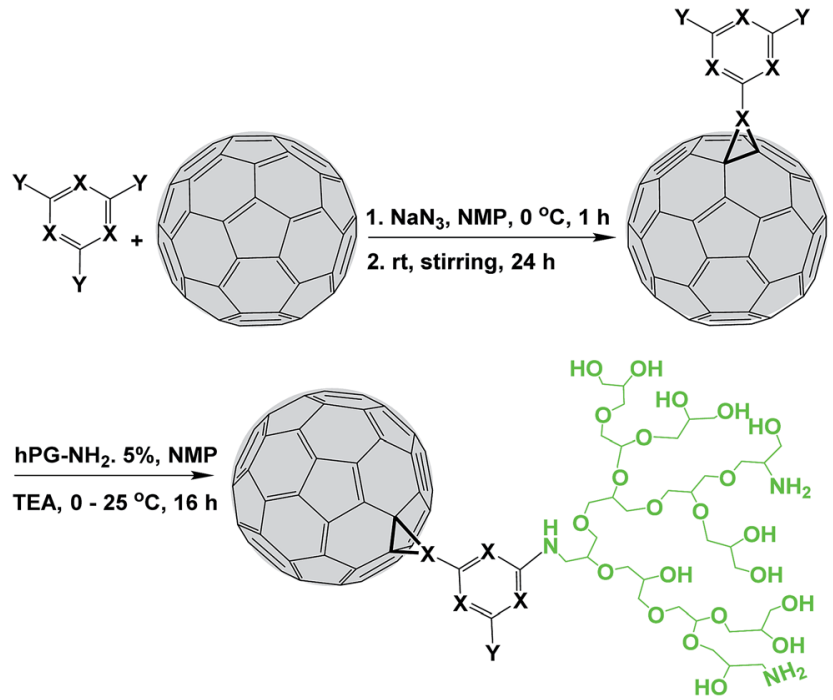

Scheme 1 Functionalization of fullerene by an in situ [2 + 1] nitrene cycloaddition reaction at room temperature. Chlorine and nitrogen atoms are symbolized by $Y$ and $X$, respectively.

fullerene $\left(\mathrm{C}_{\mathrm{Full}}\right)$ at $284.6 \mathrm{eV}$, an intense peak at $286.3 \mathrm{eV}$, which is assigned to the carbon atoms of functional groups $\left(\mathrm{C}_{\mathrm{Trz}}\right)$, provides another evidence for conjugation of triazine to the surface of fullerene (Fig. 1a). The peak area ratio of these two types of carbon atoms $\left(\mathrm{C}_{\mathrm{Trz}} / \mathrm{C}_{\mathrm{Full}}\right)$ is 0.56 , confirming that FullTrz consists of $56 \%$ triazine and $44 \%$ fullerene moieties (Table S1 $\dagger$ ). Such ratio indicates conjugation of 5 triazine units to the surface of fullerene.

The N K-edge NEXAFS spectrum of Full-Trz at the low-energy side (Fig. 1b) reveals two sharp resonances (I, II) that correspond with triazine groups that are attached to fullerene. The low energy resonance (I) is related to the nitrogen atoms in the triazine ring which was found in NEXAFS spectra of triazine in the gas phase. ${ }^{15,16}$ Resonance (II) also is characteristic for triazine molecules, coupled to carbon materials and was reported in literature. ${ }^{\mathbf{1 3}}$

In the IR spectra of Full-Trz, two absorbance bands, respectively located at 1181 and $1427 \mathrm{~cm}^{-1}$ are the characteristic peaks of $\mathrm{C}_{60}$ (Fig. S2a $\dagger$ ). ${ }^{17}$ Besides, the absorbance bands at 1510 and $1634 \mathrm{~cm}^{-1}$ are assigned to $\mathrm{C}=\mathrm{N}$ and $\mathrm{C}=\mathrm{C}$ bonds of the triazine
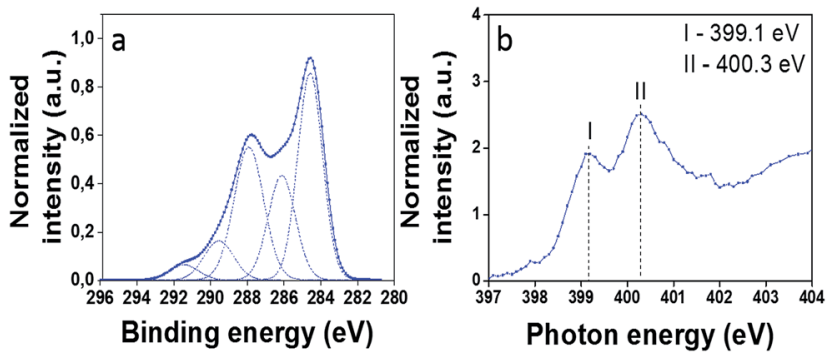

Fig. 1 (a) Highly resolved XPS spectra with peak fitting for C 1s Full-Trz, (b) expanded low energy section of N K-edge NEXAFS of Full-Trz. For assignments see Tables S1 and S2.† and the fullerene fragments, respectively. In the IR spectra, the characteristic bands of the aziridine rings, created by the $[2+1]$ cycloaddition reaction, usually appear around 1000 and 1250 $\mathrm{cm}^{-1}$. Those bands are due to symmetric aziridine ring breathing modes and to the $\mathrm{sp}^{3}$-hybridized $\mathrm{C}-\mathrm{N}$ bonds. ${ }^{18}$ The absence of such absorbance bands in the IR spectra of Full-Trz could reflect the opening of the aziridine ring and subsequent formation of an azafulleroid structure.

Furthermore, the number of triazine functional groups conjugated to the surface of fullerene was also calculated by thermogravimetric analysis (TGA). Based on the mass loss at $480{ }^{\circ} \mathrm{C}$, we found that the total mass of triazine groups in FullTrz is $55 \%$. This indicates that 5 triazines conjugate to one $\mathrm{C}_{60}$ molecule (Fig. S3†). This result is in good agreement with XPS data. The UV-vis spectrum of Full-Trz shows a broad absorption band in the 200-800 $\mathrm{nm}$ range (Fig. $2 \mathrm{a}$ ). The extension of the UV absorption of fullerene from short wavelengths $(200-430 \mathrm{~nm})$ to near-IR is due to the substantial changes in its $\pi$-conjugated system.

Usually, covalent functionalization of carbon based nanomaterials by $[2+1]$ cycloaddition reaction results in $\mathrm{sp}^{3}$ carbon atoms (at the functionalization site) with consequent disruption of the $\pi$-conjugation system. ${ }^{19}$ As a result, the useful optoelectronic properties of carbon based nanomaterials will be suppressed, which is a hampering factor for their future applications. $^{20}$ In contrast, the present functionalization improve the intrinsic optochemical properties of fullerene, significantly.

The mechanism we propose, as an explanation for these noticeable observations, implies the opening of the aziridine rings followed by $\mathrm{sp}^{3} \rightarrow \mathrm{sp}^{2}$ rehybridization of the carbon atoms (Fig. 3).

This type of isomerisation, usually referred to as aziridinofullerene-azafulleroid interconversion, has been reported for fullerene derivatives previously. ${ }^{10,11}$ However, much harder reaction conditions were used.

A similar isomerisation has been observed for single walled carbon nanotubes functionalized by carben ${ }^{21,22}$ or $[2+1]$ nitrene cycloaddition reaction. ${ }^{\mathbf{1 1}}$ There is a strong correlation between the curvature of tubes and this type of isomerization. ${ }^{21}$ Therefore, one would expect that this conversion is more feasible for fullerene as a highly curved aromatic system. Accordingly,
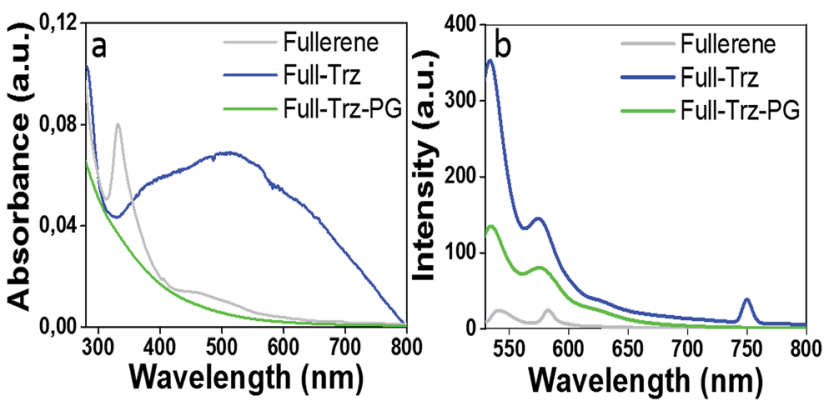

Fig. 2 (a) and (b) UV and fluorescence spectra of $0.1 \mathrm{mg} \mathrm{ml}^{-1}$ solution of fullerene (in DMF), Full-Trz (in PBS) and Full-Trz-PG (in PBS), respectively. Spectra are recorded at room temperature. 

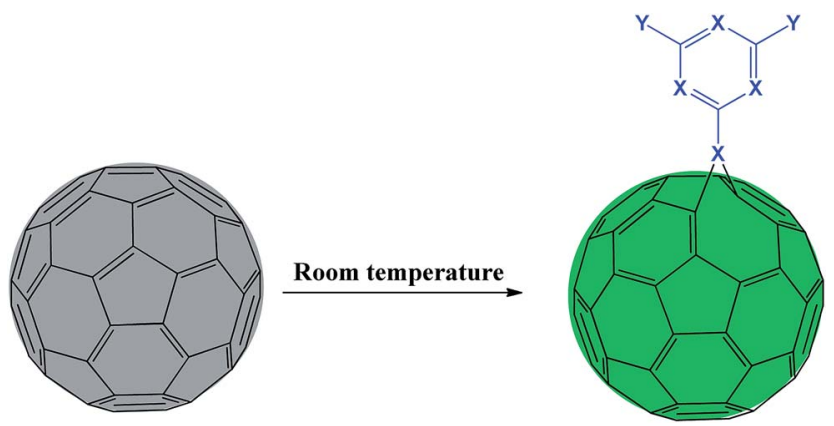

Fig. 3 Aziridinofullerene-azafulleroid interconversion and rehybridization of the carbon atoms of fullerene at ambient condition. $X$ and $Y$ are nitrogen and chlorine atoms, respectively.

computational studies were performed to investigate Full-Trz isomerization.

While aziridinofullerene-azafulleroid interconversion requires heating,,$^{23,24}$ the functionalization of fullerene by triazine in 5,6/5 and 5,6/6 positions returns preferentially azafulleroid structures (Fig. 4). Moreover, the relative energy of the frontier orbitals of the pristine and functionalized fullerene was calculated. Interestingly, the HOMO-LUMO separation in the azafulleroid form was found to be very close to the one calculated for the pristine $\mathrm{C}_{60}$ (Table $\mathrm{S} 3 \dagger$ ).

Given the fact that even a very low number of $\mathrm{sp}^{3}$ carbons would determine a dramatic decrease in the HOMO-LUMO gap of fullerene, ${ }^{25}$ the computational results strongly uphold the hypothesis of a retained, homogeneous $\mathrm{sp}^{2}$ hybridization of all carbon atoms in Full-Trz.

In the open azafulleroid form, the $\pi$-conjugated system of fullerene is regenerated and the $\pi$-network extends over the triazine rings (Fig. 4). As a result a broad band appears in the absorption spectra of the Full-Trz.

Optical properties of fullerene derivatives were also investigated computationally (ESI page 9 and $10 \dagger$ ). When triazine was arranged in a symmetric shell around fullerene, the UV spectra of Full-Trz was very similar to the experimental data (Fig. S6 and S7 $\dagger$ ). These results suggest not only a core-shell structure for the functionalized fullerene but also prove the influence of the triazine shell on the optical properties of fullerene. a

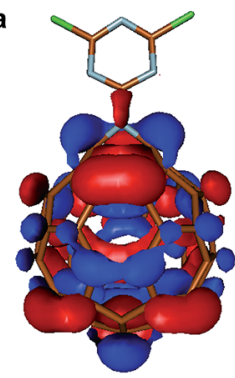

b

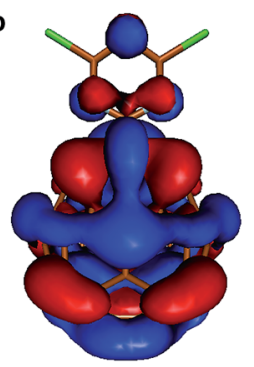

Fig. 4 Representation of the HOMO of (a) 5,6/5-Full-Trz and (b) 5,6/ 6-Full-Trz calculated at the M06-2X/def2-SVP level of theory, using an isosurface value of 0.02 e $\AA^{-3}$.
The near-IR absorption suggests that Full-Trz should exhibit unique optical properties that are observed for single walled carbon nanotubes.

Irradiation of a $5 \mathrm{mg} \mathrm{ml}^{-1}$ dispersion of Full-Trz in PBS increase the temperature of medium to $53{ }^{\circ} \mathrm{C}$ after 60 seconds, while this effect was not observed for fullerene when exposed under the same conditions (Fig. 5).

Due to such high photothermal conversion, Full-Trz could be used for variety of applications such as photothermal therapy. Interestingly, Full-Trz shows a high fluorescence emission upon excitation at different wavelengths (Fig. S4 $\dagger$ ). Therefore, it could be used as an alternative for single-wall carbon nanotubes and in particular for bioimaging.

However, poor water solubility of Full-Trz hampers such applications. Therefore, polyglycerol with few amino functional groups was conjugated to Full-Trz at room temperature, taking advantage of the high reactivity of triazine groups toward nucleophilic reactions (Scheme 1). IR spectrum of fullerenepolyglycerol conjugate (Full-Trz-PG) shows absorbance bands at 3365,1652 and $1100 \mathrm{~cm}^{-1}$ which are assigned to $\mathrm{O}-\mathrm{H}, \mathrm{C}=\mathrm{C}$ and $\mathrm{C}-\mathrm{O}$ bonds. This result confirms conjugation of polyglycerol to $\mathrm{C}_{60}$ (Fig. S2 $\dagger$ ). Thermogravimetric analysis (TGA) shows that Full-Trz-PG is consisting of $90 \%$ polyglycerol and $10 \%$ fullerene moieties ${ }^{5 d}$ (Fig. S3†). This compound shows photothermal and fluorescence properties similar to Full-Trz and it is readily soluble in PBS (Fig. 2, 5 and S8†).

While the fullerene content of Full-Trz-PG is low (10\%), its fluorescence is remarkable. Therefore it can be considered as a promising vector with the unique optoelectronic properties for bioimaging (Fig. S9†).

Quantum yields of Full-Trz and Full-Trz-PG are much higher than those reported for fullerene (Table $S 4 \dagger$ ).

In order to investigate the efficiency of Full-Trz and Full-TrzPG as fluorescence probes, they were incubated with the A549 cells and then imaged by confocal laser scanning microscopy (CLSM). Fig. 6 show confocal images of A549 cells incubated with these compounds after 4 hours. Uptake of both Full-Trz

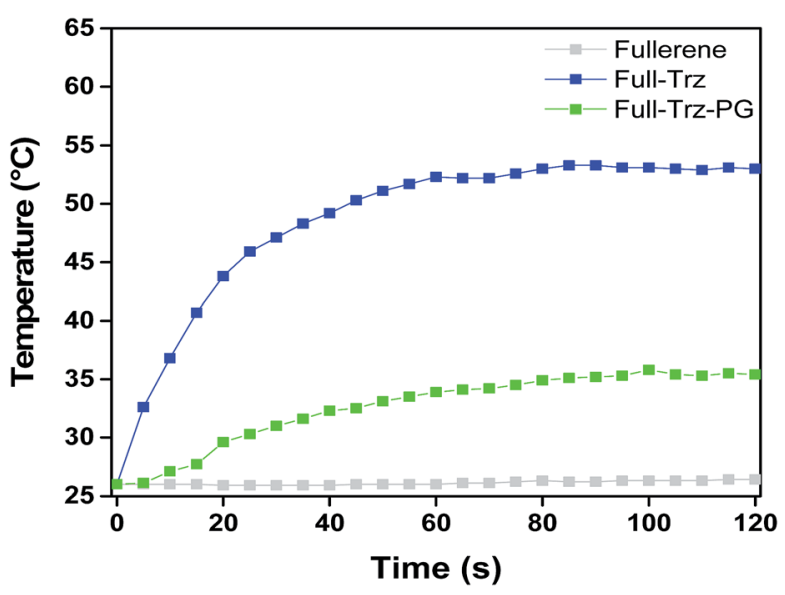

Fig. 5 Evaluation of photothermal conversion of fullerene, Full-Trz, and Full-Trz-PG dispersions in PBS $\left(5 \mathrm{mg} \mathrm{ml}^{-1}\right)$ over a period of $2 \mathrm{~min}$ under NIR laser irradiation $\left(785 \mathrm{~nm}, 0.5 \mathrm{~W} \mathrm{~cm}^{-2}\right)$. 

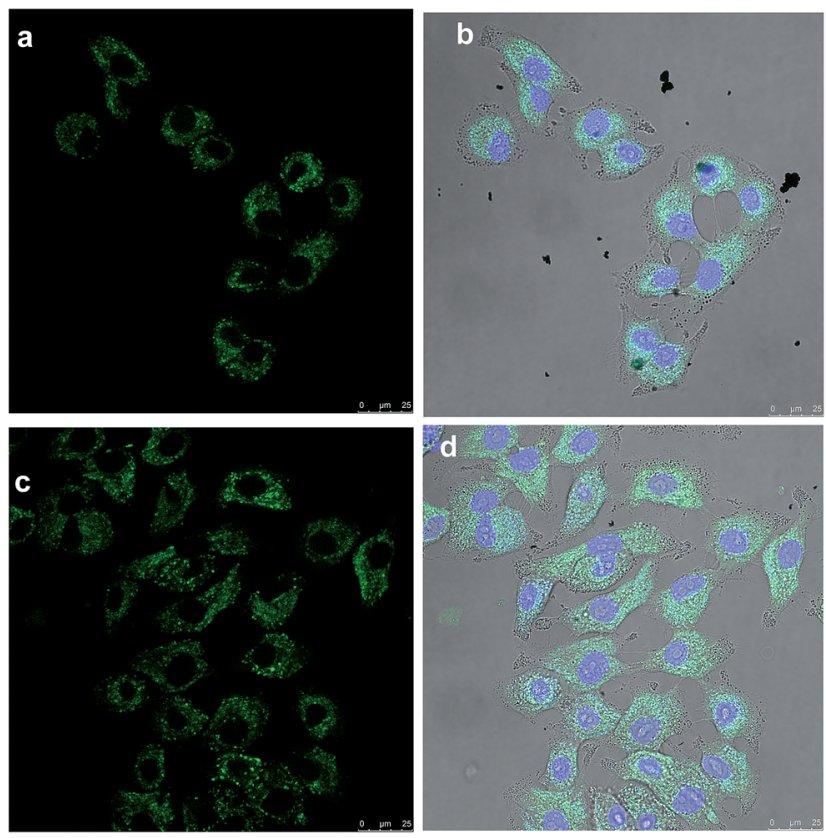

Fig. 6 The CLSM images of A549 cells incubated with $30 \mu \mathrm{g} \mathrm{ml}^{-1}$ of Full-Trz (a, b) and Full-Trz-PG (c, d) after 4 hours. Left column is fluorescence images of Full-Trz (up) and Full-Trz-PG (down) and right column is the related overlay images.

and Full-Trz-PG can be followed through their fluorescence. However, intensity of fluorescence in the case of Full-Trz-PG is higher, considering 10\% Full-Trz content for this compound. Due to the higher solubility of Full-Trz-PG and less aggregation in buffer lower quenching of fluorescence occurs.

Taking advantage of this new functionalization method, fullerene derivatives with the unique optoelectronic and physicochemical properties for a variety of applications could be synthesized. Due to its well-defined structure, Full-Trz is good alternative to nanomaterials with less-defined structures such as single walled carbon nanotubes and quantum dots in bioimaging.

\section{Acknowledgements}

The authors would like to thank the SFB 765 for financial support.

\section{Notes and references}

1 (a) E. E. Maroto, M. Izquierdo, S. Reboredo, J. MarcoMartínez, S. Filippone and N. A. Martín, Acc. Chem. Res., 2014, 47, 2660-2670; (b) M. D. Tzirakis and M. Orfanopoulos, Chem. Rev., 2013, 113, 5262-5321; (c) S.-E. Zhu and G.-W. Wang, Chem. Soc. Rev., 2013, 42, 75357570; (d) S. S. Babu, H. Möhwald and T. Nakanishi, Chem. Soc. Rev., 2010, 39, 4021-4035.

2 (a) R. Bakry, R. M. Vallant, M. Najam-ul-Haq, M. Rainer, Z. Szabo, C. W. Huck and G. K. Bonn, Int. J. Nanomed., 2007, 2, 639-649; (b) P. Compain, C. Decroocq, J. Iehl,
M. Holler, D. Hazelard, T. M. Barragán, C. O. Mellet and J.-F. Nierengarten, Angew. Chem., Int. Ed., 2010, 49, 57535756; (c) M. Durka, K. Buffet, J. Iehl, M. Holler, J.-F. Nierengarten, J. Taganna, J. Bouckaert and S. P. Vincent, Chem. Commun., 2011, 47, 1321-1323.

3 (a) J. M. Ashcroft, D. A. Tsyboulski, K. B. Hartman, T. Y. Zakharian, J. W. Marks, R. B. Weisman, M. G. Rosenblum and L. J. Wilson, Chem. Commun., 2006, 28, 3004-3006; (b) T. Y. Zakharian, A. Seryshev, B. Sitharaman, B. E. Gilbert, V. Knight and L. J. Wilson, J. Am. Chem. Soc., 2005, 127, 12508-12509; (c) R. D. Bolskar, Fullerenes for drug delivery, in Encyclopedia of Nanotechnology, ed. B. Bhushan, Springer, Netherlands, 2012, pp. 898-911.

4 (a) S. Minakata, R. Tsuruoka and M. Komatsu, J. Am. Chem. Soc., 2008, 130, 1536-1537; (b) Y. Matsuo, K. Kanaizuka, K. Matsuo, Y.-W. Zhong, T. Nakae and E. Nakamura, J. Am. Chem. Soc., 2008, 130, 5016-5017; (c) C. Yang, J. Y. Kim, S. Cho, J. K. Lee, A. J. Heeger and F. Wudl, J. Am. Chem. Soc., 2008, 130, 6444-6450.

5 (a) C.-L. He, R. Liu, D.-D. Li, S.-E. Zhu and G.-W. Wang, Org. Lett., 2013, 15, 1532-1535; (b) S. Afreen, K. Muthoosamy, S. Manickam and U. Hashim, Biosens. Bioelectron., 2015, 63, 354-364; (c) A. Muñoz, D. Sigwalt, B. M. Illescas, J. Luczkowiak, L. Rodríguez-Pérez, I. Nierengarten, M. Holler, J.-S. Remy, K. Buffet, S. P. Vincent, J. Rojo, R. Delgado, J.-F. Nierengarten and N. Martín, Nat. Chem., 2016, 8, 50-57; (d) I. Donskyi, K. Achazi, V. Wycisk, C. Böttcher and M. Adeli, Chem. Commun., 2016, 52, 43734376.

6 J. Averdung and J. Mattay, Tetrahedron, 1996, 52, 54075420.

7 L. Ulmer and J. Mattay, Eur. J. Org. Chem., 2003, 2933-2940. 8 J. Jeong, M. Cho, Y. T. Lim, N. W. Song and B. H. Chung, Angew. Chem., Int. Ed., 2009, 48, 5296-5299.

9 J. Jeong, J. Jung, M. Choi, J. W. Kim, S. J. Chung, S. Lim, H. Lee and B. H. Chung, Adv. Mater., 2012, 24, 1999-2003.

10 T. Nakahodo, M. Okada, H. Morita, T. Yoshimura, M. O. Ishitsuka, T. Tsuchiya, Y. Maeda, H. Fujihara, T. Akasaka, X. Gao and S. Nagase, Angew. Chem., Int. Ed., 2008, 47, 1298-1300.

11 H. Hachiya, T. Kakuta, M. Takami and Y. Kabe, J. Organomet. Chem., 2009, 694, 630-636.

12 A. Setaro, M. Adeli, M. Glaeske, D. Przyrembel, T. Bisswanger, G. Gordeev, F. Maschietto, A. Faghani, B. Paulus, M. Weinelt, R. Arenal, R. Haag and S. Reich, 2016, Submitted.

13 D. Sheppard, R. Terrell and G. Henkelman, J. Chem. Phys., 2008, 128, 134106.

14 G. Henkelman, B. Uberuaga and H. Jónsson, J. Chem. Phys., 2000, 113, 9901-9905.

15 E. Apen, A. P. Hitchcock and J. L. Gland, J. Phys. Chem., 1993, 97, 6859-6866.

16 G. Vall-llosera, B. Gao, A. Kivimaeki, M. Coreno, J. A. Ruiz, M. de Simone, H. Agren and E. Rachlew, J. Chem. Phys., 2008, 128, 139901.

17 K. Saeedfar, L. Y. Heng, T. L. Ling and M. Rezayi, Sensors, 2013, 13, 16851-16866. 
18 H. L. Spell, Anal. Chem., 1967, 39, 185-193.

19 M. L. Usrey, E. S. Lippmann and M. S. Strano, J. Am. Chem. Soc., 2005, 127, 16129-16135.

20 L. Cognet, D. A. Tuyboulski, J.-D. R. Rocha, C. D. Doyle, J. M. Tour and R. B. Weisman, Science, 2007, 316, 14651468.

21 Y.-S. Lee and N. Marzari, Phys. Rev. Lett., 2006, 97, 116801.
22 K. Zhang, Q. Zhang, C. Liu, N. Marzari and F. Stellacci, Adv. Funct. Mater., 2012, 22, 5216-5223.

23 M. Cases, M. Duran and M. Solà, J. Mol. Model., 2000, 6, 205212.

24 K. Raghavachari, Chem. Phys. Lett., 1993, 209, 223-228.

25 H. Tachikawa, T. Iyama and S. Abe, Phys. Procedia, 2011, 14, 139-142. 